\title{
Ontologies-based architecture for sociocultural knowledge co-construction systems
}

\author{
Guidedi Kaladzavi, University of Maroua, Maroua, Cameroon, kaladzavi@univ-maroua.cm \\ Papa Fary Diallo, University of Gaston Berger, Saint-Louis, Senegal, diallo.papa- \\ fary@ugb.edu.sn \\ Cedric Bere, University of Ouagadougou, Burkina Fasso, cedric.bere@gmail.com \\ Olivier Corby, Université Côte d'Azur, Sophia, France, olivier.corby@inria.fr \\ Isabelle Minrel, Université Côte d'Azur, Sophia, France, isabelle.mirbel@unice.fr \\ Moussa Lo, University of Gaston Berger, Senegal, moussa.lo@ugb.edu.sn \\ Kolyang, University of Maroua, Maroua Cameroon, kolyang@univ-maroua.cm
}

\begin{abstract}
Considering the evolution of the semantic wiki engine on-based platforms, two main approaches could be distinguished: Ontologies for Wikis (OfW) and Wikis for Ontologies (WfO). OfW vision requires the existing ontologies to be imported. Most of them use the Resource Description Framework (RDF-based) systems in conjunction with the standard Structured Query Language (SQL) database to manage and query semantic data. But, relational database is not an ideal type of storage for semantic data. A more natural data model for Semantic MediaWiki (SMW) is $R D F$, a data format that organizes information in graphs rather than in fixed database tables. This paper presents an ontology on-based architecture, which aims to implement this idea. The Architecture mainly includes three-layered functional architecture: Web User Interface Layer, Semantic Layer, and Persistence Layer.
\end{abstract}

Keywords: Ontologies, knowledge management systems, architecture, co-construction, semantic wikis.

\section{Introduction}

This research study is set in an African context, where the main problem is economic, social development, and the means to achieve it. Indeed, after the failure of the several development models in the recent decades, theoretical research seems to be turning to the development knowledge-based approaches (UNESCO, 2014). The place of knowledge, science, and technology in the current dynamics of growth gives rise to intensify the reflection within the economic field. In particular, many authors pointed out the start of a new phase of knowledgebased development following a phase of the physical capital accumulation.

To promote the indigenous knowledge, some media could be used: a permanent (re)education, the radio broadcasting, and off course the Internet, which seems to be the best media (UNESCO, 2014). It reduces instantaneously distances between civilizations. Thus, it is an opportunity to disseminate the local knowledge on a large scale. But, this is not sufficient to make the Internet 
the ultimate solution to the African culture vulgarization. It does not create anything itself. It is the African responsibility to build the content of the "empty shell" that is the Internet and make the rational use, while enjoying the opportunities it offers.

To get there, new computational technologies, i.e. semantic technologies, are needed to manage these large repositories of sociocultural data, while discovering some useful patterns and knowledge from them. Semantic Web vision proposed by Tim Berners-Lee, revolutionized the Web architecture. The Web architecture switched from the documentary graph to the published as well as interconnected databases with capabilities to "understand" their semantics and reason on them. Technically, the Resource Description Framework (RDF) data model and the Uniform Resource Identifier (URI) standard for modeling as well as identifying resources on the Web were introduced into the Web architecture stack. As result, the Web is spreading in the World and the World is spreading in the Web with issues such as cultural "digital divide". Indeed, a cultural void in the Web of Data is the lack of that culture at the applicative level (e-tourism, etc.) of the Web of Data.

In this paper, we present the architecture of the sociocultural knowledge co-construction platforms, developed to enable some communities to share and co-build their cultural heritage. Thus, the architecture relies essentially on the Upper-Level Sociocultural Ontology (USCO) (Diallo, Corby, Mirbel, Lo, \& Seydina, 2014) ontology, which allows communities to share and co-construct their sociocultural knowledge. Since the USCO ontology is aligned with the vocabularies of Schema.org and DBpedia, the latter are also integrated and used in the platform. To allow temporal annotation of resources, the Human Temporal Ontology (HuTO) ontology is used (Diallo, Corby, Mirbel, Lo, \& Seydina, 2015). Ontoshare models how contributors could co-build the content of the Knowledge Management System (KMS) circumscribed by USCO (Kaladzavi, Diallo, Kolyang, \& Lo, 2015). The functionalities of the platform could also allow members of the African communities, Senegalese in particular, to collaborate knowledge, exchange, and compare their points of view in the process of co-construction of knowledge.

The rest of the paper is schemed as follows. In Section II, we present the definition of some core concepts. Section III entitled related work, reviews some existing studies devoted to the Sociocultural Knowledge Management. Then, Section IV depicts the proposed architecture. The Architecture presentation consists of presenting its main layers. In Section V, we point out the performance evaluation feedback. The paper ends with a conclusion and future trends.

\section{Conceptual Framework}

We present in this section the definition of some core concepts, which could facilitate the understanding of the paper.

\section{Culture}

Culture consists of a complex whole, which includes knowledge, belief, art, morals, law, customs, as well as any other capabilities and habits acquired by humans as a member of a society (Tylor, 2005). There are many definitions of culture depending on the domain, which as in philosophy, sociology, etc. In sociology, the definition of culture is more narrow, and refer to "what is common to a group of individuals" (Dressen-Hammouda, 2012, p. 510) that is to say 
what is learned, transmitted, produced, and created. For United Nations for Educational, Scientific and Cultural Organization (UNESCO), culture may now be regarded as the set of "distinctive spiritual, material, intellectual and emotional features that characterize a society or social group" (UNESCO, 2001, para 5). Culture encompasses the arts, literature and science, lifestyles, fundamental rights of the human being, value systems, traditions, as well as beliefs (UNESCO, 1982).

\section{Sociocultural Knowledge}

The sociocultural qualifier links the belief, art, morals, law, custom, as well as any other capabilities and habits to a society. In other words, the qualifier links to a group of people (European, African, Asian, etc.) who shares the same experiences. Sociocultural knowledge describes information about society and culture. It is related to, or involves, a combination of social and cultural factors. It acquires its richness in an environment where several people share, a space (continent, country, region, state, \& locality) in which there are interactions between cultures.

Being interested by the sociocultural knowledge in the sense of its valorization and transmission to the future generations, is to focus on the heritage aspects of a society, a people or a country. Heritage territories are plural and fuzzy (Benhamou, \& Thesmar, 2011). Since 1986, Chastel already mentioned that the concept of heritage is global, vague, and pervasive (Benhamou \& Thesmar, 2011). Some experts, along with the public, defined heritage territories as concept that is intended to be preserved. It is, therefore, subject to possible redefinitions. Then, the heritage perimeter is drawn by UNESCO, which establishes criteria and a list of heritages. According to UNESCO, heritage means, that a country intends to preserve for the future generations. It includes both a report on the history and the future, because of its continuities with the benefit of hindsight that brings almost naturally new monuments in the heritage field. Moreover, it includes discontinuities with the introduction of new objects and concepts that expand the field of heritage, gardens, landscapes, industrial sites, public infrastructures (schools, airports, hospitals, etc.), various memorial sites, etc. However, it also includes intangible heritage such as the traditional festivities, sport activities, religious activities, etc. that contribute to the development of what could be called a 'national romance.'

\section{Ontologies}

The foundational definition of ontology is proposed by Gruber (1993) as "an explicit specification of a conceptualization" (p. 1). The exact meaning depends on the understanding of the terms 'specification' and 'conceptualization'. According to Genesereth and Nelson (1987), conceptualization is a "set of objects, concepts, and other entities that are presumed to exist in some domains of interest and the relationships that hold them" (p. 1). In the Gruber (1993)'s definition, it's not clear that specification depends on the logical view of ontologists. That is why Guarino and Giaretta (1995) introduced the logical theory instead of mere specification (Camara, 2013). Afterward, Borst (1997) enriched the previous definition by adding consensual facts related to knowledge modeling discipline characteristics such as sharing and reuse (Camara, 2013). For Borst (1997), "Ontologies are defined as a formal specification of a shared conceptualization” (p. 12). Finally, Studer, Benjamins, and Fensel (1998) merged the existing 
definitions to note: "An ontology is a formal and explicit specification of a shared conceptualization. They underline the necessity of formal, explicit and shared paradigms" ( $p$. 184). Even if, it's the merging of the existing definitions, it seems consensual. It's more cited in recent years, demonstrating its compliance with the expectations of the Knowledge Base Systems designers (Camara, 2013). The explicitly, formality, and shareability of knowledge features in ontology are carried out by five elements: concepts, relations, functions, axioms, and instances (Gomez-Pérez, 1999).

\section{Co-Construction}

The term co-construction is an innovative term, which burst into everyday language recently: used in print media once a year before 2003, once a month in 2005, it appears almost daily in 2013 (Akrich, 2013). Typically, it is used to enhance the involvement of the plurality of actors in the development and implementation of a project or an action. In the academic literature, the term has undergone a parallel evolution to that, which is observed in the press. In Educational Sciences, the term co-construction denotes the desire to get out of a vertical transmission of knowledge by actively and collaboratively involving pupils or students in the production of learning content through Information and Communication Technologies (ICT). In sociology or political science, the term means the existence of the plurality of actors involved into the production of a policy, project, a technical device or knowledge. In the case of HumanComputer-Interaction (HCI), co-construction implements how users interact among themselves to collaboratively achieve a virtual activity through software interface. It is the fundamental idea behind the Web 2.0 vision, which democratizes the knowledge production on the Web. By Web 2.0, Internet users are no longer just consumers but also authors of information. Thus, the social Web enables Internet users to share knowledge that is to externalize their expertise about something or to learn (internalize) what other Internet users have published.

\section{Related Work}

Since the Web 2.0, the flows have been reversed: the user is no longer passive (reader) but active (author). The transition from Web 1.0 to Web 2.0 done in 2004 was a decisive articulation to the social knowledge management systems (i.e. social media). Social media includes all tools and applications that allow interaction between Internet users. Within that galaxy of social media, there are several 'planets'. Out of them, there are texts publishing tools (wiki, blogs, etc.), exchange and sharing tools (YouTube for videos, Slideshare for sharing presentations, etc.), tools for discussion (Skype, Messenger, WhatsApp, etc.) and the networking implementation tools (Twitter, Facebook, Myspace, etc.). Unfortunately, most of them are not local-knowledge oriented. Some cases exist, such as Wikipedia and Afripedia. Afripedia is a project devoted to develop Wikipedia in the African context. It is the main project launched in 2012 by the Agence Universitaire de la Francophonie (AUF), the French Institute (IF), and the French Wikimedia Association, which aimed to Africanize the Wikipedia encyclopedia by integrating the African knowledge in the platform, even where the Internet access is not possible (that means in the offline way). In fact, before the Afripedia project, Wikimedia association was worried about the few contributions from Africa and relative to Africa. In geographic sections for example, it is even came to see that a mountain somewhere in African locality is described as a hill. In 
addition, the platform does not use semantic technologies, which means it is not ontology based (kaladzavi et al., 2015).

The management of knowledge is increasingly being recognized as a key element in extracting its value. Knowledge Management (KM) is a discipline that provides strategy, process, and technology to share as well as leverage information and expertise that will increase the level of understanding to (more) effectively solve problems as well as make decisions (Satyadas Harigopal, \& Cassaigne, 2001). KM role can be viewed as turning data into information and then forming information into knowledge. It is largely regarded as a cyclic process involving various activities (Nonaka, 2007). The process can be subdivided, for example into creating internal knowledge, acquiring external knowledge, storing knowledge, as well as updating the knowledge and sharing knowledge internally or externally (Alavi \& Leidner, 2001). The KMS architecture is a fundamental issue in the area of $\mathrm{KM}$ that must be well resolved in order to deliver competitive services to the users and the organization. Meso and Smith (2002) proposed a KMS architecture that processes a combination of all the aspects stated below as well as other components, which is able to perform according to the requirement of the organization. These components consist of technology functions and the knowledge itself. Different factors and constraints influence the development of an architecture: functional requirements, quality considerations, experience, and technical aspects (Jacobson, Booch, \& Rumbaugh, 1999). In the context of Web of Data, the architecture is primarily influenced by functional requirements, which means the services provided and the quality considerations such as scalability, performance, reusability, and semantic interoperability. These constraints are the foundations for data models. Thus, the semantic layer compound by RDF, Resource Description Framework Schema (RDFS), Rule Interchange Format (RIF), Semantic Web Rule Language (SWRL), Standard Protocol and RDF Query Language (SPARQL), and Web Ontology Language (OWL) technologies are key components in the architecture design.

\section{Architecture}

We proposed in our previous work (Diallo et al., 2014; Diallo et al., 2015; Kaladzavi et al., 2015) how we can create, acquire, store, as well as update and share the sociocultural knowledge internally and externally by modeling some sociocultural ontologies:

- USCO: USCO ontology is used to enable communities to share and co-build their sociocultural heritage. This is done through the descriptions made of the individuals of sociocultural events organized by these communities, the descriptions of the available resources to these communities but also the descriptions made about these communities themselves.

- HuTO: HuTO models the deictic dates, which are dates that form a specific relation with the time of the discourse. In our modeling, deictic times are sub-concepts of the Date concept. Indeed, one cannot know what refers to a deictic date without knowing the temporal position of the discourse. The modeling choice is to model the deictic time by associating the properties of the effective date with the deictic time. 
- Ontoshare: Ontoshare is a virtual activity ontology in the case of the sociocultural knowledge sharing. It designs how Internet users could share and co-construct the content of a sociocultural KMS.

The developed architecture (See Figure 1) is based on three entities: 1) Semantic MediaWiki (SMW), which is the base of the platform, 2) Virtuoso, which is the triple store (RDF database), and Exhibit, which is the data visualization tool. Figure 1 shows the different layers of the architecture. This architecture consists of three layers: A Human-Machine-Interface (HMI) supported by SMW and Exhibit. This layer allows users to participate in the co-construction of knowledge. It also makes it possible to present the data in a user-friendly way (Exhibit). The semantic layer makes it possible to have a query interface and a rules engine. The persistence layer consists of a relational database and a triple store (semantic database).

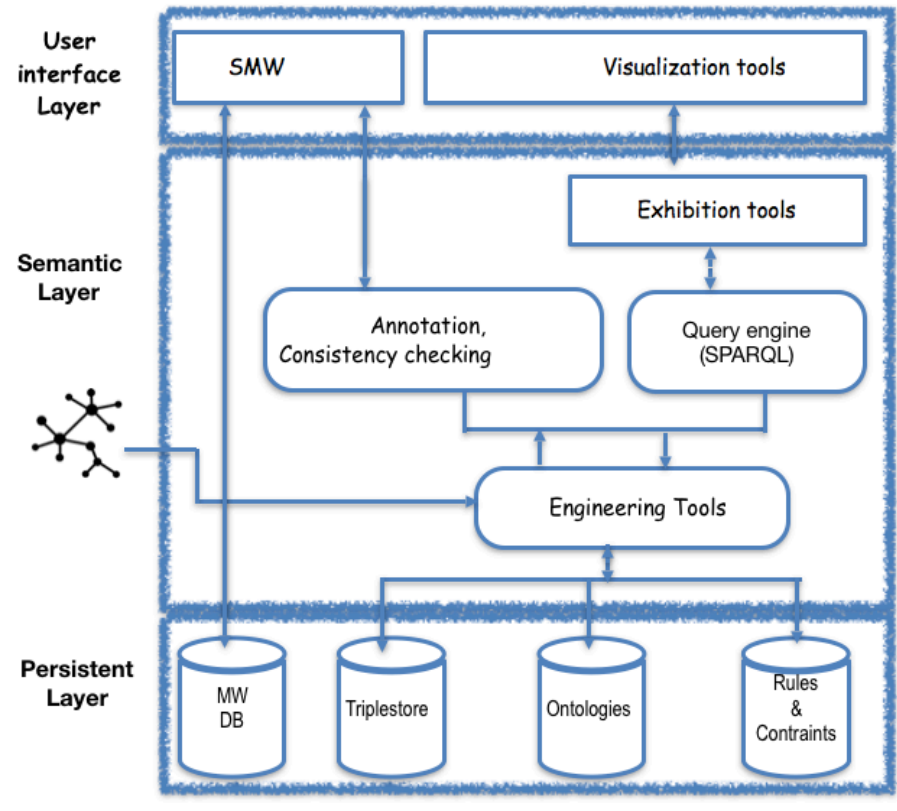

Figure 1. System Architecture

\section{Web User Interface Layer}

The HMI layer is composed of the implemented tools, so that a human can control and communicate with the platform. The first tool is SMW, an extension of MediaWiki (MW). The second tool is Exhibit, which belongs to the Semantic Interoperability of Metadata and Information in unLike Environments (SIMILE) project (MIT, 2006).

\section{Semantic MediaWiki (SMW)}

SMW is an extension of MW, the Wikipedia-based wiki engine (semantic-mediawiki.org, 2017). Unlike traditional wikis that can only contain texts that can be not 'understood' or evaluated by software agents, SMW adds semantic annotations to the Wiki pages using an extension of the MW script language. Thus, SMW enables to MW, function as a collaborative knowledge base. MW has a scripting language to describe the content of the Wiki pages. This language was 
extended by SMW with the following three sets of semantic annotations: Classes, Properties, Axioms, and Instances. Thus, the collection of semantic data in SMW is made by allowing users to add annotations on the articles pages through the special tags. Each item corresponds exactly to an ontological element, that is to the following RDF types: owl:Thing, rdfs:Class, owl:ObjectProperty, owl:DatatypeProperty, and owl:AnnotationProperty. In particular, an article is an instance of owl:NamedIndividual, categories become classes and properties depending on their type in the Wiki (See Table 1).

Table 1. Mappings between SMW and OWL

\begin{tabular}{|l|l|l|}
\hline SMW & Syntaxis & OWL \\
\hline Relation & {$[$ [ Capital::Senegal] $]$} & owl:ObjectProperty \\
\hline Attribute & {$[$ [ Population::1056009] $]$} & owl:DatatypeProperty \\
\hline Category(in article) & {$[$ [ Category:City] $]$} & owl:NameIndividual \\
\hline Category(in a namespace) & $[$ [ Captegory::Locality $]]$ & Rdfs:subClassOf \\
\hline
\end{tabular}

\section{Exhibit}

Exhibit is developed during the SIMILE project, a collaboration between Massachusetts Institute of Technologies (MIT), the Artificial Intelligence Lab (CSAIL), and the World Wide Web Consortium (W3C). The goal of the SIMILE project is to develop tools to increase the interoperability of disparate digital collections and develop robust open source tools that allow users to access, manage, visualize as well as reuse schemas/vocabularies/ontologies and metadata (MIT, 2006). Exhibit is part of these tools and allows creating Web pages with a support of sorting, filtering, and rich visualizations by writing only Hypertext Markup Language (HTML), some Cascading Style Sheets (CSS), and JavaScript codes. The Exhibit user interface consists of three main components types: Collection, Widget, and Coder.

\section{Semantic Layer}

The main role of the semantic layer is to link the Knowledge Base: 1) to Exhibit for better visualization, 2) to the external resources to feed the Knowledge Base, and 3) to the defined rules to deduce implicit relations in the Knowledge Base. All these actions are done through the Jena framework. It provides an Application Program Interface (API) for retrieving and writing data in RDF graphs. The graphs are represented as an abstract model. A template can come from data, files, databases, URIs, or a combination of them. A model can also be queried by a SPARQL Endpoint. Thus, to allow access to the data stored in Virtuoso, the triplestore provides a Jena RDF Data Provider, which is a service for the Jena framework and allows Semantic Web applications to directly query a Virtuoso Knowledge Base. Query Engine (See Figure 1) is a SPARQL Endpoint interface provided by Virtuoso. This interface allows users to directly access Virtuoso data. However, thanks to the Jena framework, Web applications are developed to access 
the data of the platform. Rules Engine is a Java Enterprise Edition (EE) component that is developed and that executes these rules. However, we used in these rules the INSERT clauses of SPARQL in place of the CONSTRUCT clauses.

\section{Persistence Layer}

Typically, SMW stores data in a Relational Database. However, for RDF data, the best option is to use a data format that organizes information in the form of triples rather than in relational database tables. Indeed, the use of a triplestore has several advantages:

1. Better performance for information retrieval: $\mathrm{RDF}$ databases are configured to respond to the SPARQL queries, which is a W3C recommendation. SPARQL offers better performance in information retrieval than the use of SQL queries in a relational database. Indeed, the SPARQL queries make it possible to benefit from all the semantics of the RDF data.

2. An additional interface: with a SPARQL Endpoint (Web query interface), it's possible to make SPARQL queries on the data without resorting to the interface of SMW. This allows other applications to access the data. A reasoning functionality: semantic Web languages such as RDFS and OWL offer powerful modeling features such as the possibility of wording subsumption relations between the concepts or characteristics of certain properties as transitivity. Thus, an RDF database can infer on these characteristics to get answers. The reuse of ontologies and the integration of data: it's possible to add the external data in the RDF database and to use SMW to update them. Thus, the RDF database can be seen as a platform for data integration and ontologies reuse.

3. Physical separation of resources: Separating the data used by MW from SMW provides a way to distribute tasks across multiple servers. In particular, complex queries, which consume a large amount of computational power, thus, may not affect the basic operation of the Wiki. Therefore, SMW can operate with five different triple blinds. Table 2 compares these triple blinds. A graph database represents the data in visualized structures composed of nodes and edges. This allows easy processing of the data by a calculation of the specific properties of the graph, such as the number of steps (nodes) needed to go from one node to another. A triplestore implements a graph model that interprets the predicate as the label of a link between subject and object.

Among the triplestores (see Table 2), Virtuoso has been used. It is a hybrid database and a middleware that supports relational data, graphical and RDF data, Web-server applications, and so on. By integrating Virtuoso with SMW, only the semantic data is migrated into Virtuoso. As shown in Figure 1, all textual data remains in the MySQL database that is used by MW, and all annotations made in the Wiki are stored in Virtuoso. For the better scaling, the database is divided into three warehouses (graphs): 1) a warehouse where the semantic data are stored (annotations from the Wiki), 2) a warehouse where the USCO ontology is stored, and 3) a warehouse where the HuTO ontology is stored. Since SMW allows importing vocabularies, the vocabularies of HuTO and USCO are imported into the platform. This allows users to annotate the resources of the Wiki using the vocabularies of HuTO, USCO, and Ontoshare. 
Table 2. Comparison of the Triplestores Used by SMW

\begin{tabular}{|l|c|c|c|c|c|}
\cline { 2 - 6 } \multicolumn{1}{l|}{} & Blazegraph & Jena TDB & Sesame & Virtuoso & 4store \\
\hline Supported Model & $\begin{array}{c}\text { Graphic } \\
\text { and RDF } \\
\text { store }\end{array}$ & $\begin{array}{c}\text { RDF } \\
\text { stores }\end{array}$ & $\begin{array}{c}\text { RDF } \\
\text { stores }\end{array}$ & $\begin{array}{c}\text { Graphic } \\
\text { And } \\
\text { RDF stores }\end{array}$ & $\begin{array}{c}\text { RDF } \\
\text { stores }\end{array}$ \\
\hline $\begin{array}{l}\text { Storable triple } \\
\text { stores }\end{array}$ & $12.7 \mathrm{~B}$ & $1.7 \mathrm{~B}$ & $70 \mathrm{M}$ & $15.4 \mathrm{~B}$ & $15 \mathrm{~B}$ \\
\hline $\begin{array}{l}\text { RDF store } \\
\text { classification }\end{array}$ & 15 & 3 & 4 & 2 & 8 \\
\hline \\
* - 'M' stands for million and 'B' stands for billion \\
** For example, DBpedia has less than 440M triplets
\end{tabular}

Performance Evaluation

\title{
Semantic Interoperability
}

The proposed architecture enables the use of the external resources to feed the Knowledge Base. These resources could be imported from the Linked Open Data, open data for which, a SPARQL Endpoint interface exists. The English version of DBpedia was used to extract data related to Senegalese case study. Indeed, DBpedia proposes a Knowledge Base that gathers several themes and data specific to countries. Thus, the alignment between the DBpedia concepts such as DBpedia:PopulatedPlace and usco:Locality enables to extract all Senegalese cities from the Knowledge Base of DBpedia using Query 1.

\author{
PREFIX ontology: <http://dbpedia.org/ontology/> \\ PREFIX resource: $<$ http://dbpedia.org/resource/> \\ SELECT * \\ WHERE \{ ?1 ontology:type resource:Regions_of_Senegal . \\ ?o ontology:isPartOf ?1 \}
}

Query 1. Request to have the regions of Senegal and their administrative subdivisions that are in the Knowledge Base of DBpedia.

\section{Rules}

HuTO provides a conceptual model in RDFS for modeling temporal expressions and annotating RDF resources. However, many temporal relationships are implicitly expressed in occurrences of events (relative dating). Answers to many time-oriented questions are not necessarily explicitly represented but can be deduced. To do this, it proposed a set of rules to standardize the 
representation of temporal data and also rules of inference. Since HuTO is an ontology in RDFS, some rules have been proposed, expressed as CONSTRUCT queries in SPARQL, with the aim of deducing and explaining the maximum temporal information to enable the reasoning capabilities. The temporal information can be expressed in different ways. For example, a date (month) can be represented by using the calendar representation (See Query 2). Also, some rules have been created to standardize these types of writings. Therefore, whatever the writing used mode, possible representations will be added to the data graph.

PREFIX huto: $<$ http://ns.inria.fr/huto/>

CONSTRUCT \{ ?x huto:number ?m ; huto:numberOfDay ?d ; huto:even ?e\}

WHERE \{

$$
\begin{aligned}
& \text { ?x rdf:type ?o } \\
& \text { ?o rdfs:subClassOf huto:Month ; } \\
& \text { huto:number ?m ; } \\
& \text { huto:even ?e }
\end{aligned}
$$

OPTIONAL $\{$ ?x rdf:type/huto:numberOfDay ?d\}

Query 2. Example of month normalization rules.

\section{Inferences}

However, since RDFS does not implement some basic inferences such as transitivity or reflexivity, we created some inference rules for this purpose. Thus, we defeated inference rules for the transitivity of before/after properties. Similarly, if a relation (before or after) is expressed between two events (respectively intervals), it is necessary to propagate this relation between the intervals (respectively resources) concerned. For this, we proposed propagation rules. In total, 26 rules of implications and inferences were defined for properties before and after. These rules are defined for transitivity, inverse and propagation (See Query 3).

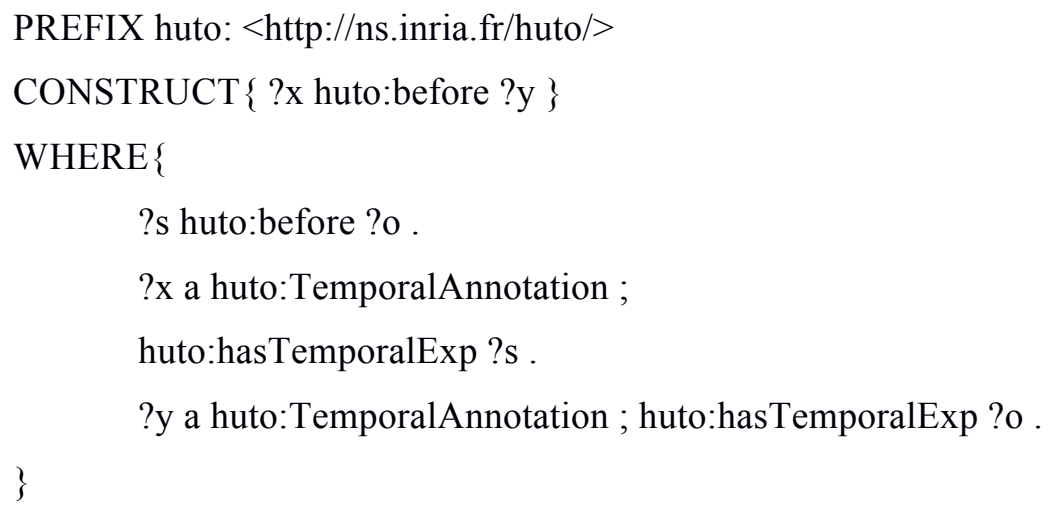

Query 3. Rule for propagating the before property between resources. 


\section{Semantic Queries}

We distinguish two types of queries: 1) A resource-type request: determines the period of occurrence of the given resource, and 2) Typical queries on temporal elements: they determine resources relating to a given period of occurrence or relative to a temporally annotated resource. The SPARQL query (See Query 4) is an example of the request written from the resource-type request in which, the retrieved resource was specified. It allows to determine the temporality of the resource data:Gamou.
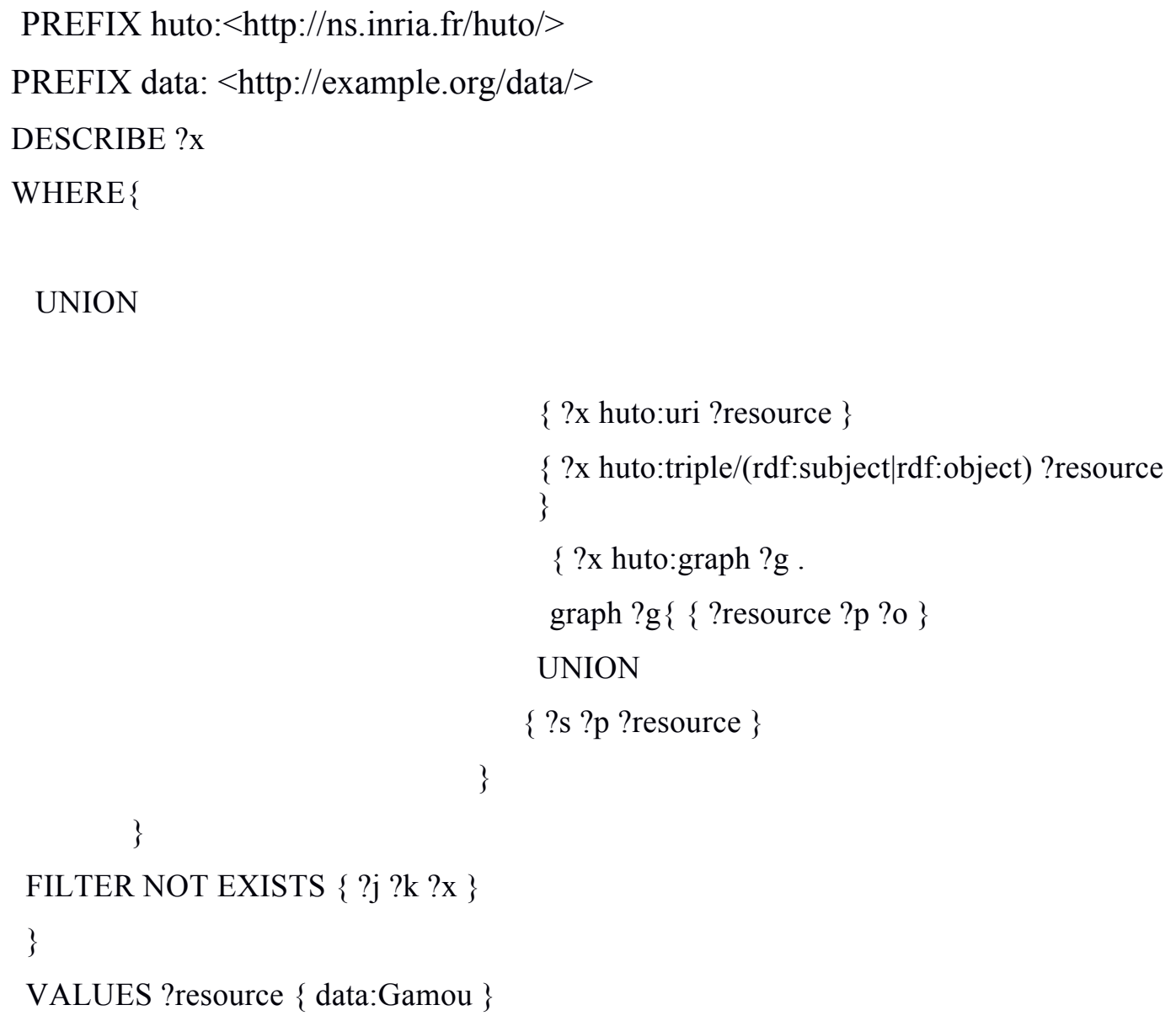

Query 4. Determining the temporality of the resource data:gamou

\section{Conclusion and Perspectives}

In this paper, we presented an ontology-based architecture for the sociocultural KMS. The platform can be considered as a "collective memory" that enables users to share and co-build knowledge. The platform could help to capture the holistic view of the local changes while considering culture and historicity in the context of a country. The proposed architecture includes three layered functional architecture: Web user interface layer, semantic layer, and persistent 
layer. We illustrated how Internet users can internalize or externalize knowledge in the ethical way in the Senegalese context. In addition, we evaluated the architecture performance regarding semantic interoperability, rules, inferences features. The Success of the system depends on some ICT features. That's why we would like to point out that some ICT access features such as: " divide by access ", " divide by decision " in African Countries must be improved. Collaborative knowledge base systems face the speculations problems. Yet, the credibility of contributors is increasingly sought after by fact-checking algorithms. As result, the future work will be focused on how to take into account the fact-checking module in our architecture.

\section{Acknowledgement}

In memory of Papa Fary Diallo, who brutally left us while we were discussing on this paper. The authors would like to thank the Centre d'Excellence Africain en Mathématiques (CEA-MITIC), Informatique et TIC, the University of Maria (UMa), and Laboratoire d'Analyse Numérique et d'Informatique (LANI) for the financial support.

\section{References}

Akrich, M. (2013). Co-construction. Dictionnaire critique et interdisciplinaire de la participation. Retrieved from http://www.participation-et-democratie.fr/es/dico/coconstruction

Alavi, M., \& Leidner, D. E. (2001). Knowledge management and knowledge management systems: Conceptual foundations and research issues. MIS Quarterly, 25(1), 107-136.

Benhamou, F., \& Thesmar, D. (2011). Valoriser le patrimoine culturel de la France. Technical report, UNESCO.

Borst, W. (1997). Construction of engineering ontologies for knowledge sharing and reuse (Unpublished doctoral dissertation). University of Twente, Netherlands.

Camara, G. (2013). Conception d'un système de veille épidémiologique à base d'ontologies: Application à la schistosomiase au Senegal. Ph.D. dissertation, Université Pierre MarieCurie \& Université Gaston Berger, Saint-Louis.

Diallo, P. F., Corby, O., Mirbel, I., Lo, M., \& Seydina, M. N. (2014). Sociocultural ontology: Upper level and domain ontologies. Proceedings of JFO, Hammamet, Tunisia, 15-27.

Diallo, P. F., Corby, O., \& Mirbel, I., Lo, M., \& Seydina, M. N. (2015). HuTO vocabulary specification. Retrieved from http://ns.inria.fr/huto

Dressen-Hammouda, D. (2012). Ethnographic approaches in ESP. In B. Paltridge, S. Starfield (eds.), The handbook of English for Specific Purposes (pp. 501-518). Oxford, UK: WileyBlackwell Publishers.

Genesereth, M. R., \& Nilsson, N. J. (1987). Logical foundations of artificial intelligence, Los Altos, CA: Morgan Kaufmann Publishers Inc. 
Gomez-Pérez, A. G. (1999). Overview of knowledge sharing and reuse components: Ontologies and problem-solving methods. Proceedings of the IJCAI-99 workshop on Ontologies and problem-Solving Methods (KRR5), Stockholm, 111-115.

Gruber, T. R. (1993). A translation approach to portable ontology specifications. Knowledge Acquisition Journal, 5(2), 199-220.

Guarino, N., \& Giaretta, P., (1995). Ontologies and knowledge bases: Towards a terminological clarification. In Mars, N. J. I. (Ed.), Towards Very Large Knowledge Bases: Knowledge Building and Knowledge Sharing (pp. 25-32). Amsterdam, Natherlands: IOS Press.

Jacobson, I., Booch, G., \& Rumbaugh, J. (1999). The unified software development process. Boston, MA: Addison-Wesley.

Kaladzavi, G., Diallo, P. F., Kolyang, \& Lo, M. (2015). Virtual activity ontology modeling: the case of sociocultural knowledge sharing. Proceedings of the Fifth International Conference on Social Media Technologies, Communication, and Informatics, Barcelona, 36-41.

Meso, P., \& Smith, R. (2002). A resources-based view of organizational knowledge management systems. Journal of Knowledge Management, 4(3), 224-234.

MIT (2006). Exhibit: Publishing framework for data-rich interactive Web pages. Retrieved from http://www.simile-widgets.org/exhibit/

Nonaka, I. (2007). The knowledge-creating company. Retrieved from https://hbr.org/2007/07/ the-knowledge-creating-company.

UNESCO (1982). Déclaration de mexico sur les politiques culturelles. Technical Report. Retrieved from: http://www.culture.gouv.fr/Media/Thematiques/Egalite-et-diversite/Colle ge-de-la-Diversite/Declaration-de-Mexico

UNESCO (2001). UNESCO universal declaration on cultural diversity. Retrieved from: http://portal.unesco.org/en/ev.php-URL_ID=13179\&URL_DO=DO_TOPIC\&URL_SECT $\underline{\mathrm{ION}}=201 . \mathrm{html}$

UNESCO (2014). Edifier des sociétés de savoirs inclusives. Technical Report. Retrieved from: http://unesdoc.unesco.org/images/0022/002279/227938f.pdf

Satyadas, A., Harigopal, U., \& Cassaigne, N. P. (2001). Knowledge management tutorial: An editorial overview. IEEE Transactions on Systems, Man, and Cybernetics-Part C: Applications and Review, 31(4), 429-437.

Semantic-mediawiki.org (2017). Semantic MediaWiki (SMW). Retrieved from https://www.semantic-mediawiki.org/wiki/Semantic_MediaWiki

Studer, R., Benjamins, R., \& Fensel, D. (1998). Knowledge engineering: Principles and methods. Journal of Data \& Knowledge Engineering, 25(4), 161-197.

Tylor, E. B. (2005). Primitive culture: Researches into the development of mythology, philosophy, religion, art, and custom. New York, NY: Gordon Press. 


\section{Authors' Biographies}

Guidedi Kaladzavi is a Lecturer and researcher in Computer Engineering at the University of Maroua, Cameroon. He received his Ph.D. degree in Computer Science in 2016. Since 2017, he has been a postdoctoral researcher in WIMMICS team, INRIA Sophia Antipolis in the MoReWAIS (Mobile Read Write Access and Intermittent to Semantic Web) project under the Co-supervision of Moussa LO and Fabien Gandon. His current research is focused on Ontology engineering, Web of Data, Mobile Semantic Web.

Papa Fary Diallo. He received his Ph.D. degree in computer science in 2016. He was a postdoctoral researcher at the African Center of Excellence (www.ceamitic.sn) of the University of Gaston Berger, Saint-louis, Senegal and in WIMMICS team, INRIA Sophia Antipolis in the MoReWAIS (Mobile Read Write Access and Intermittent to Semantic Web) project under the Co-supervision of Moussa LO and Fabien Gandon.

Cedric Bere is an Assistant-lecturer at the University of Ouaga 1 Professeur Joseph KI-ZERBO. $\mathrm{He}$ is a PhD student at the University of Gaston Berger, Senegal. His current research is focused on the Ontology Engineering.

Olivier Corby is an Inria Researcher, Wimmics team of University of Côte d'Azur, I3S, CNRS, SPARKS. He received his $\mathrm{PhD}$ in Computer Science at the University of Nice - Sophia Antipolis. His research topics are Semantic Web, Linked Data \& Knowledge Representation and Reasoning. He has a special interest in Graph based Knowledge Representation, RDF/S, SPARQL.

Isabelle Minrel is an Associate Professor, University of Nice-Sophia Antipolis UINRIA Sophia Antipolis, France. Member of the WIMMICS research team I3S (CNRS-UNS) / INRIA. She has a special interest in the Semantic Requirement Engineering.

Moussa Lo is a Professor of Computer Science at the University of Gaston Berger, Senegal and Head of the IT team at the Laboratory of Numerical Analysis and Computing (LANI), he was a leader of UFRSAT, then Coordinator of the CEAMITIC. Since April 1999, he has been conducting research activities in the 2SIWeb2 group (Information System for the Web) of the University of Pau and Pays de l'amour, under the direction of Mr. Amrane HOCINE. Currently, he is the Coordinator of the Virtual University of Senegal. His current research is focused on semantic Web, Integration, mobile Web, Web of Data.

Kolyang is a Professor of Computer Science at the University of Maroua, Cameroon. He received his $\mathrm{PhD}$ at the University of Bremen, Germany in 1997 on Formal Methods in Software Engineering under the supervision of Prof. Dr. KriegBrückner. In 2008 he got a Habilitation The- sis at the University of Bremen. Since 2015 he has been nominated Research Ambassador of the University of Bremen, Germany. Since 2015, he is also Expert of CAMES for distance and open learning, for the higher education systems in Africa. Currently, his research domains focus on Software. 